\title{
Some physiological correlates of verbal learning task difficulty'
}

JOHN L, ANDREASSI

U. S. NAVAL TRAINING DEVICE CENTER

Eight Ss learned three lists of nonsense syllables (0\%, $53 \%$ and $100 \%$ association value) on three successive days while several physiological variables were recorded. Ss showed significant increases in both palmar skin conductance and heart rate with the $100 \%$ list as compared with the $53 \%$ and $0 \%$ lists. These findings were interpreted in terms of greater degrees of physiological arousal during periods of superior performance.

The relationship between skin resistance, EEG and nonsense syllable learning was studied by Obrist (1950). He found that large magnitudes of GSRs and higher frequencies of the alpha rhythm were both positively related to more efficient learning. Malmo (1965) reported that muscle potentials, heart rate and respiration were higher when Ss performed well on a pursuit rotor task than when performance was poor. The purpose of the present experiment was to explore the relationship between verbal learning, task difficulty and several physiologicál variables, viz., palmar skin conductance (PSC), heart rate (HR), galvanic skin responses (GSRs) and muscle action potentials (MAPs)。

\section{Method}

The Ss of this experiment were eight Navy enlisted men ranging in age from 22-35. All Ss were tested for $1 \mathrm{hr}$. on three consecutive days, except for one $\mathrm{S}$ who was tested on two successive days. Ss were asked to sit in a large, comfortable chair and electrodes were attached for recording purposes. Two electrodes (Telemedics, Inc., Southampton, Pennsylvania) were placed over the flexor digitorum of the right forearm to measure MAPs. The $1 \mathrm{~cm}$ diameter metal portion of the electrodes were about $4 \mathrm{~cm}$ apart. Electrode jelly was rubbed into the skin and into the fabric surrounding the metal electrode until skin resistance was below $5,000 \mathrm{ohms}$. HR was measured by placing one electrode on the bicep of the right arm and another on the left forearm. Two Yellow Springs Instrument electrodes were clamped to the palm of the left hand to measure skin resistance. The electrodes were $2 \mathrm{~cm}$ diameter zinc plates mounted in plastic cups and filled with zinc sulphate paste. A ground electrode was attached to the left ear lobe and connected to ground on the Offner terminal board, as were shielded leads for HR and MAPs. After it was ascertained that all recordings were being satisfactorily obtained, $S$ was asked to "relax as though you were going to sleep."

Subsequent to obtaining an initial resting level for each of the physiological measures, $S$ was presented with a practice list which consisted of $73 \%$ association value nonsense syllables (Hilgard, 1951). S practiced the anticipation method of serial learning for $5 \mathrm{~min}$. (this corresponded to eight presentations of the list) and then rested. The experimental lists consisted of 16 syllables each and difficulty level was estimated according to association values which were $0 \%, 53 \%$ and $100 \%$ (Hilgard, 1951). Syllables were presented at a 2 sec. rate. After each list $S$ had a 2 min. resting period resulting in a total of five measured resting periods for each session. The presentation of lists was counterbalanced so that each $\mathrm{S}$ began each session with a different list. Each of the lists, both practice and experimental, was presented for eight trials on each day.

Thirty sec. samples of physiological measures were taken for all trials, i.e., each presentation of a list, and for rest periods. The number of heart beats during the 30-sec. trial period were recorded with an Elmeg Printer. Skin resistance and GSRs were recorded directly with an Offner Skin Resistance Coupler Type 9892A. A line of best fit was judged visually to determine the average skin resistance value during each trial and reciprocals of these values were multiplied by one million to obtain conductance units (micromhos). Skin resistance changes of greater than $1,000 \mathrm{ohms}$ within a 2-sec. period were designated as GSRs.

Results

The average HR for the eight Navy men increased with increasing association value (see Fig. 1). This rise was statistically significant for two of the comparisons $(0 \%$ vs. $100 \%$ association value at $p<.01$, and $100 \%$ vs. $53 \%$ at $\mathrm{p}<.05$ ). A two-tailed criterion for statistical

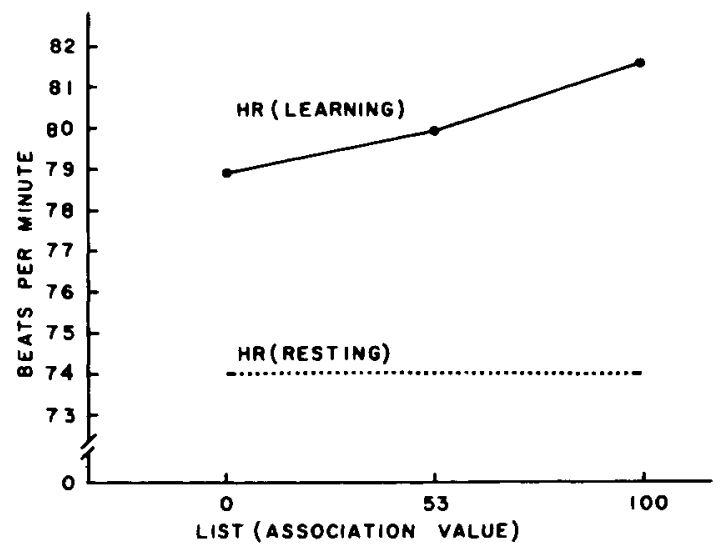

Fig. 1. Mean HR during learning and resting conditions. 


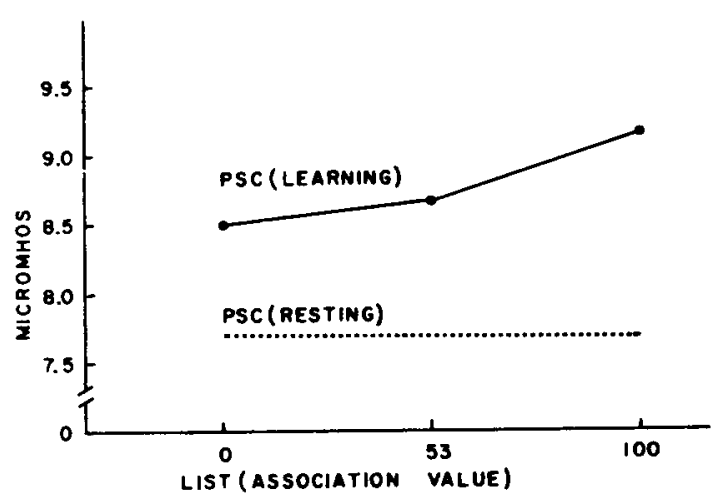

Fig. 2. llean PSC during learning and resting conditions.

significance was used in all tests. The resting $H R$ level shown in Fig. 1 is not associated with any particular list since it was attained by averaging the values for all rest periods over the three experimental sessions. The same procedure was used to obtain the resting levels for the other physiological measures.

The mean PSC data also show a rise with ease of list (see Fig. 2). This rise was statistically significant for two of the comparisons $10 \%$ vs. $100 \%$ at $\mathrm{p}<.01$, and $100 \%$ vs. $53 \%$ at $p<.05)$. The mean number of GSRs which occurred during each learning trial indicate a slight but non-significant increase in number of GSRs for the easier lists (see Fig. 3). Since there was evidence that external noise was included in the MAP records, these data will not be presented. There were significant differences in learning rate for all comparisons, i.e., $0 \%$ vs. $53 \%, 0 \%$ vs. $100 \%$ and $53 \%$ vs. $100 \%$, at $\mathrm{p}<.01$.

\section{Discussion}

The Navy men became moreactivated physiologically with the easier learning materials, perhaps because

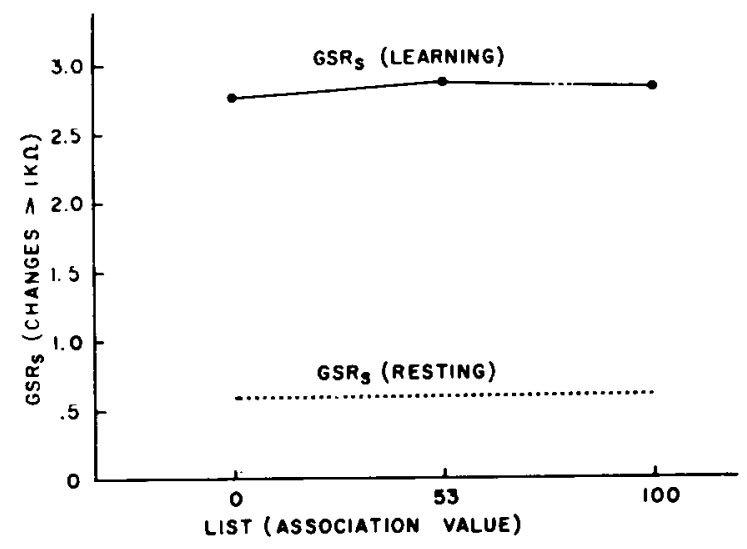

Fig. 3. Mean GSRs during learning and resting conditions. they anticipated more syllables correctly with the easier materials. In other words, Ss may have become more involved in the learning task when their performance was more successful. The type of increased physiological activity shown in the present experiment has been found in past studies to be related to such things as speed and accuracy of performance in mirror tracing (Bartoshuk, 1955) and increases in incentive (Stennett, 1957). In Bartoshuk's study there were increases in MAPs while in Stennett's study MAPs and PSC both showed increases. Obrist (1950) concluded that learning is associated with a high degree of arousal which is greatest when the greatest amount of learning takes place. Thus, the findings of past studies and the present study indicate that motivational factors could lead to greater degrees of physiological arousal during periods of superior performance. This possibility is supported by some observational data on the one $S$ out of eight whose physiological responses did not vary with list difficulty. This $\mathrm{S}$ seemed indifferent towards the experiment, his learning record was poorest and he was the only $S$ to tall asleep during one of the two min. rest periods. Perhaps this $S$ was not involved in the task because even the easier lists were too difficult for him. Other Ss may have lost interest with the more difficult lists and this could be a possible explanation for their lower level of physiological arousal with the more difficult materials.

The number of GSRs showed no difference between the three lists. Perhaps GSR frequency, representing a measure which is sensitive to so many stimuli, may not be capable of yielding differential response to subtle differences such as list difficulty.

\section{References}

Bartoshuk, A. K. Electromyographic gradients in goal directed activity. Canad. J. Psychol., 1955, 9, 21-28.

Hilgard, E. R. Methods and procedures in the study of learning. In S. S. Stevens (Ed.), Handbook of experimental psychology. New York, Wiley, 1951, 517-567.

Malmo, R. B. Physiological gradients and behavior. Psychol. Bull., $1965,64,225-234$.

obrist, W. D. Skin resistance and electroencephalographic changes associated with learning. Unpublished doctoral dissertation, Northwestern University, 1950.

Stennett, R. G. The relationship of performance level to level of arousal. J. exp. Psychol., 1957, 54, 54-61.

\section{Note}

1. This paper represents a portion of the following report: Andreassi, J. L., \& Cavallari, J. D. Biopotential signals as a function of learning task difficulty. NAVTRADEVCEN Tech. Rept. IH-34, 1965. 